\title{
Micromagnetic Dissipation, Dispersion, and Mode Conversion in Thin Permalloy Platelets
}

\author{
M. Buess, ${ }^{1,2}$ T. Haug, ${ }^{1}$ M. R. Scheinfein, ${ }^{3}$ and C. H. Back ${ }^{1}$ \\ ${ }^{1}$ Institut für Experimentelle und Angewandte Physik, Universität Regensburg, Universitätsstrasse 31, 93040 Regensburg, Germany \\ ${ }^{2}$ Laboratorium für Festkörperphysik, Eidgenössische Technische Hochschule Zürich, CH-8093 Zürich, Switzerland \\ ${ }^{3}$ Simon Fraser University, 8888 University Drive, Burnaby BC V5A 1S6, Canada
}

(Received 8 November 2004; published 1 April 2005)

\begin{abstract}
Micron-sized ferromagnetic Permalloy disks exhibiting an in-plane ferromagnetic vortex structure are excited by a fast rise time perpendicular magnetic field pulse and their modal structure is analyzed. We find azimuthal and axial modes. By a Fourier filtering technique we can separate and analyze the time dependence of individual modes. Analysis of the experimental data demonstrates that the azimuthal modes damp more quickly than the axial modes. We interpret these results as mode conversion from lowfrequency azimuthal modes to the fundamental mode which is higher in frequency, i.e., mode-mode coupling in a system with a single Landau-Lifshitz-Gilbert phenomenological damping constant $\alpha$.
\end{abstract}

DOI: 10.1103/PhysRevLett.94.127205

The time scales for ultrafast magnetic processes can be loosely divided into the femtosecond regime where single electron and optical excited ions are probed (see $[1,2]$ and references therein) and the picosecond regime where magnetization dynamics are governed by the Landau-LifshitzGilbert (LLG) equations. In the LLG regime, optical [3-7] and soft $\mathrm{x}$-ray $[8,9]$ pump-probe experiments are employed to examine magnetization dynamics excited by short magnetic field pulses, typically triggered by a laser pulse. Time dependent measurements in the LLG regime are nearly ideal for determining the modal frequencies, the mode structure [3,5,7], and the phenomenological damping parameter $[6,10,11]$ which can be directly compared to measurements using ferromagnetic resonance (FMR) $[12,13]$. When measurements of the damping parameter extracted from quasilinear time-domain measurements do not agree with FMR measurements [10,14], explanations typically invoke sample and field inhomogeneities [14,15] and higher order magnon scattering. In order to probe damping mechanisms typical of the intrinsically nonlinear LLG regime, we examine modes excited in lithographically produced Permalloy circular platelets [5]. Micron-sized disks are ideal systems to study mode excitation, mode conversion, and damping since the small size of the disks provides many well defined excitations [5,16-18] within the frequency range easily characterized by stroboscopic time resolved scanning Kerr microscopy [3-7]. In order to characterize the excited spin wave spectrum, we employ a phase sensitive Fourier transformation (FT) technique with windowing functions [5]. Frequency domain analysis of the time sequenced images reveals resonances corresponding to the modes defined by the boundary conditions as fixed by the shape of the elements. As the time-domain signal is Fourier transformed and linearly Fourier filtered, backtransformation at resonance provides a mode-bymode reconstruction with the spatial resolution provided by the experiment - typically $300 \mathrm{~nm}$ for Kerr microscopy. Using this technique, we have identified radially symmetric and nonaxially symmetric azimuthal modes $[5,18]$ ex-
PACS numbers: 75.30.Ds, 75.75. $+\mathrm{a}, 76.50 .+\mathrm{g}$

cited in circular Permalloy platelets with an equilibrium vortex configuration. Here we examine mode dispersion and energy channeling between modes, which leads to an apparent increase in damping for certain modes.

Our experimental analysis is performed on the stroboscopic time resolved scanning Kerr microscopy data of a single $4 \mu \mathrm{m}$ diameter, $15 \mathrm{~nm}$ thick, Permalloy disk. In the ground state the magnetization forms a vortex structure [19]. Following excitation using a perpendicular magnetic tipping pulse, polar Kerr data are recorded at 40 ps intervals. These data are images encompassing a field of view of $6.5 \mu \mathrm{m}$ square. A typical trace of the polar Kerr signal as a function of time averaged over the whole element is shown in Fig. 1(a) (left frame). Its Fourier transform is shown in Fig. 1(a) (right frame), and the observed peaks are used to identify the modes [5]. We label the excited modes using two numbers $(n, m) ; n$ counts the number of axially symmetric nodes (one node means pinning at the boundary of the element and at the vortex core) and $m$ counts the number of azimuthal nodes. We identify the fundamental $[(1,0), 3.5 \mathrm{GHz}]$, the second $[(2,0), 4.7 \mathrm{GHz}]$, and the third $[(3,0), 5.6 \mathrm{GHz}]$ radial modes possessing zero, one, and two additional radial nodes, respectively. We can also identify the dipolar $[(1,1), 2.5 \mathrm{GHz}]$ and quadrupolar $[(1,2), 2.2 \mathrm{GHz}]$ azimuthal modes which possess one and two nodes along the equilibrium direction of $\vec{M}$, respectively. For details see $[5,18]$. The Fourier spectrum does not consist of discrete sharp peaks at the modal frequencies, but of peaks with a finite width (up to $0.7 \mathrm{GHz}$ FWHM). We previously identified these excitations as eigenmodes based upon the linearized LLG equation and the scalar magnetic potential $[5,18,21]$. The peaks observed in the frequency spectrum as well as those computed from the full LLG equations are not delta functions in frequency due to the presence of dispersion, suggesting that perhaps our earlier terminology might have been misleading. Because of the finite sampling length and the resulting need of windowing, simple analysis in the frequency domain is not sufficient to illuminate the decay rates and mode 
conversion. The entire (dispersed) peak contains information about the time evolution of a given mode.

We recall briefly the relevant equations for the precession frequency for this geometry and a decay constant based on a linear, single- $\alpha$ approach: $M_{r}=M_{s} m_{r}, M_{\varphi}=$ $M_{s}\left[1-O\left(m_{\varphi}^{2}\right)\right], M_{z}=M_{s} m_{z}$, where $m_{r}$ and $m_{z}$ are the direction cosines of the magnetization perturbations, and $m_{r} \ll 1$ and $m_{z} \ll 1$. We consider thin micron-sized disks with a ground state circulating flux-closure configuration, subject to the linearized LLG equation:

$$
\begin{aligned}
& \dot{m}_{r}=\gamma_{0} m_{z} N_{z} M_{s}+\alpha \dot{m}_{z}, \\
& \dot{m}_{z}=-\gamma_{0} m_{r} N_{r} M_{s}-\alpha \dot{m}_{r} .
\end{aligned}
$$

Here, $M_{s}$ is the saturation magnetization, and $\gamma_{0}$ is the absolute value of the gyromagnetic ratio multiplied by $\mu_{0}$. $N_{z}=1$ is the demagnetizing factor for thin film shape and $N_{r}$ is defined for a given mode; for details see [5,21,22]. $N_{r}$ is proportional to the averaged effective field for that mode. Inserting oscillatory solutions $\sim e^{i \omega t}$ leads to the result for the decay time $\tau$ and the precession frequency $f$ :

$$
\tau=\frac{2\left(1+\alpha^{2}\right)}{\alpha \gamma_{0} M_{s}\left(N_{r}+N_{z}\right)}, \quad 2 \pi f \cong \gamma_{0} M_{s} \sqrt{\frac{N_{r} N_{z}}{\left(1+\alpha^{2}\right)}} .
$$



FIG. 1. Fourier filtering: Experimental data. (a) The average perpendicular magnetization component on the left and its Fourier transform on the right. (b)-(d) By applying the filters on the right before backtransformation into the time domain, the temporal evolution (circles) for different modes can be extracted. The solid lines show the fits with $M_{i}(t)=\sin \left(2 \pi f_{i} t+\varphi_{i}\right) \times$ $\exp \left(-t / \tau_{i}\right)$ (see text). $\Delta f$ is the width of the filter. (e) The lowfrequency parts up to $2 \mathrm{GHz}$ belong to the transient response on the pulse (solid line).
With $N_{z}=1$ and in the limit $\alpha \ll 1$, we obtain $\tau=$ $\frac{2}{\alpha \gamma_{0} M_{s}\left(N_{r}+1\right)}$ and $2 \pi f=\gamma_{0} M_{s} N_{r}^{1 / 2}$. Thus, to evaluate our experimental data we need to extract the experimental frequencies $f$ and the decay times $\tau$. In our implementation, the spatially averaged Kerr signal $K(t)$ is used to compute the complex Fourier spectra $S(f)$ as

$$
S\left(f_{n}\right)=\frac{1}{N} \sum_{m=1}^{N-1} K\left(t_{m}\right) H\left(t_{m}\right) e^{-2 \pi i m n / N},
$$

where $N$ is the number of data points of the temporal signal which when acquired over a fixed time interval $t$ determines the maximum Fourier frequency in the spectrum and the resolution in the frequency domain and $H(t)$ is the Hamming window function. Windowing is important for two reasons: First, the finite length of real data often has sharp transitions at the ends of the data stream, and windowing leads to a smoothing and therefore a reduction of unwanted artifacts. Second, the finite time sampling is a rectangular window, and, even a pure oscillation finitely sampled will produce side lobes in the frequency domain. The nonlinear filters $H(t)$ partially reallocate the spectral weight of the side lobes back into the associated peak. The result is a much better separation of nearby peaks at the expense of an increase in width of the main peak. Therefore, it is better to extract the damping parameter in the time domain as shown in Fig. 2 rather than from the width of the transformed peak, in contrast to FMR where the time signals are essentially of infinite duration.



FIG. 2. Fourier filtering: Micromagnetic simulation. The same modes are extracted from the computed average $M_{z}$ component as in Fig. 1. 
We can now backtransform a modal peak after applying a bandpass filter $F(f)$ to the Fourier spectra $S(f)$ since the data are ordered in frequency and the modes are obviously well separated. To make a comparison with the original temporal data, the inverse Fourier transform has to be normalized by $H(t)$ in the time domain as

$$
M_{k}\left(t_{m}\right)=\frac{1}{H\left(t_{m}\right)} \operatorname{Re} \sum_{n=1}^{N-1} S\left(f_{n}\right) F_{k}\left(f_{n}\right) e^{2 \pi i m n / N},
$$

allowing different modal components $M_{k}(t)$ of $K(t)$ in the time domain to be analyzed. If modal damping were to obey an exponential decay, an effective decay time $\tau$ for each mode can be defined. Figure 1(a) shows the averaged Kerr data $K(t)$ (left) and its Fourier transform amplitude $S(f)$ (right). The right sides of Figs. 1(b)-1(e) show the filters $F(f)_{k}$ used in Eq. (2) to obtain the modes $(1,0)$ and $(2,0)$, the azimuthal mode, and the low-frequency residuals corresponding to the modal magnetization curves $M_{k}(t)$ (left), respectively. Fits to our data using an exponentially decaying sine function (solid lines in Figs. 1(b)-1(e), left frame) lead to the decay times $\tau_{\text {exp }}^{\text {fit }}$ displayed in Table I. For the nonradially symmetric azimuthal modes, there are some peculiarities: From previous work [5,18], the peak positions for the $(1,1)$ and $(1,2)$ modes are known, and a superposition of these modes is seen in the Fourier spectrum from 2 to $2.8 \mathrm{GHz}$. This is referred to as the "azimuthal peak," and its exact appearance is determined by the spatial and temporal shape of the field pulse. The resulting value obtained by mode filtering and damped-oscillation fit is displayed as $\tau_{\text {exp }}^{\text {fit }}$. This demonstrates the significant result that the symmetry breaking azimuthal modes $\left(\tau_{\text {azim }}=\right.$ $0.37 \mathrm{~ns})$ decay 3 times faster than the fundamental mode $\left(\tau_{(1,0)}=1.1 \mathrm{~ns}\right)$ for windows whose widths $\Delta f / f_{0}$ are as good as constant ( 0.31 versus 0.37 for the azimuthal and fundamental peaks, respectively). This is a surprising result, because the calculated $\tau_{\alpha=0.008}^{\text {calc }}$ for all modes is constant within the experimental errors, as the decay constant depends weakly on $N_{r}$ in $N_{z}+N_{r}=1+N_{r} \cong 1$ [22]. Thus, additional mode damping due to energy transfer or mode conversion must occur. Furthermore, we observed despite the large error bar a significant difference for the decay of the modes $(1,0)$ and $(2,0)$. It is fortuitous to note that the higher radial modes have a very small amplitude. Consequently, the decay times of the filtered signals that have bigger errors for the modes $(2,0)$ and $(3,0)$ cannot be determined accurately. The error bar was obtained by analyzing the resulting decay time by varying the parameters of the filtering process. Unfortunately, experimentally, we cannot separate the (i) effect of the damping of each independent mode from (ii) energy that is coupled between the modes. However, there are many publications [3,5,7] that demonstrate the excellent predictive abilities of the LLG equations of motion for describing magnetization dynamics. The advantage of employing micromagnetic simulations is that energies can be monitored and global or position dependent parameters can be set. We have reproduced the details of the modal structure including the nonradially symmetric modal response [5] for excitations in circular platelets of Permalloy. Shown in Fig. 2(a) are the computed LLG $[23,24]$ averaged polar Kerr data (left) and its Fourier amplitude $S(f)$ (right). Figures 2(b)2(d) show the filters $F(f)_{k}$ used in Eq. (2) (right frame) to obtain the modes $(1,0)$ and $(2,0)$ and the azimuthal modes peak, corresponding to the modal magnetization curves $M_{k}(t)$ (left frame), respectively. At first glance one observes that in Fig. 2 the position of the resonances as well as their relative strength is very similar to the data in Fig. 1. We also observe that the decay times of the individual modes show the same trend as in Fig. 1: Fits to our data (see Table I) demonstrate the good agreement in the decay times with the experimental values. Also included in Table I are the $N_{r \text {,sim }}$ calculated by Eq. (2) from the values $f_{\text {sim }}$ and the resulting $\tau_{\alpha=0.008}^{\text {calc }}$ by Eq. (2). Again, the symmetry breaking azimuthal modes decay much faster than the fundamental mode. Finally, in the simulation it was possible to separate the azimuthal peak into the two modes. For this purpose, the average amplitude over the local Fourier transform of the magnetic movie was used to determine the separation frequency of $2.35 \mathrm{GHz}$ between the two modes. In Fig. 3 the two different filtered and backtransformed components are displayed. The resulting $\tau^{\mathrm{fit}}$ of 0.44 and $0.45 \mathrm{~ns}$ are corrected values and are $45 \%$ bigger than the decay time for the total azimuthal peak. However, it quantifies the effect of the increased decay rate by superposition of the

TABLE I. Summary of experimental and simulated results.

\begin{tabular}{|c|c|c|c|c|c|c|c|}
\hline $\begin{array}{l}\text { Mode } \\
(m, n)\end{array}$ & $\begin{array}{l}f_{\text {exp }} \\
(\mathrm{GHz})\end{array}$ & $\begin{array}{l}f_{\text {sim }} \\
(\mathrm{GHz})\end{array}$ & $N_{r, \text { sim }}$ & $\begin{array}{l}\tau_{\alpha=0.08}^{\text {calc }} \\
(\mathrm{ns})\end{array}$ & $\begin{array}{l}\tau_{\text {exp }}^{\mathrm{fit}} \\
(\mathrm{ns})\end{array}$ & $\begin{array}{l}\tau_{\text {sim }}^{\mathrm{fit}} \\
(\mathrm{ns})\end{array}$ & \\
\hline$(1,2)$ & $2.2 \pm 0.1$ & $2.21 \pm 0.05$ & 0.0053 & 1.308 & & & $0.45 \pm 0.1$ \\
\hline$(1,1)$ & $2.5 \pm 0.1$ & $2.58 \pm 0.05$ & 0.0068 & 1.306 & $0.37 \pm 0.1$ & $0.31 \pm 0.1$ & $0.44 \pm 0.1$ \\
\hline$(1,0)$ & $3.4 \pm 0.1$ & $3.41 \pm 0.05$ & 0.013 & 1.299 & $1.1 \pm 0.2$ & & $1.3 \pm 0.1$ \\
\hline$(2,0)$ & $4.7 \pm 0.1$ & $4.71 \pm 0.05$ & 0.024 & 1.283 & $0.6 \pm 0.4$ & & $1.4 \pm 0.3$ \\
\hline$(3,0)$ & $5.6 \pm 0.1$ & $5.67 \pm 0.05$ & 0.035 & 1.270 & & & $1.0 \pm 0.3$ \\
\hline
\end{tabular}



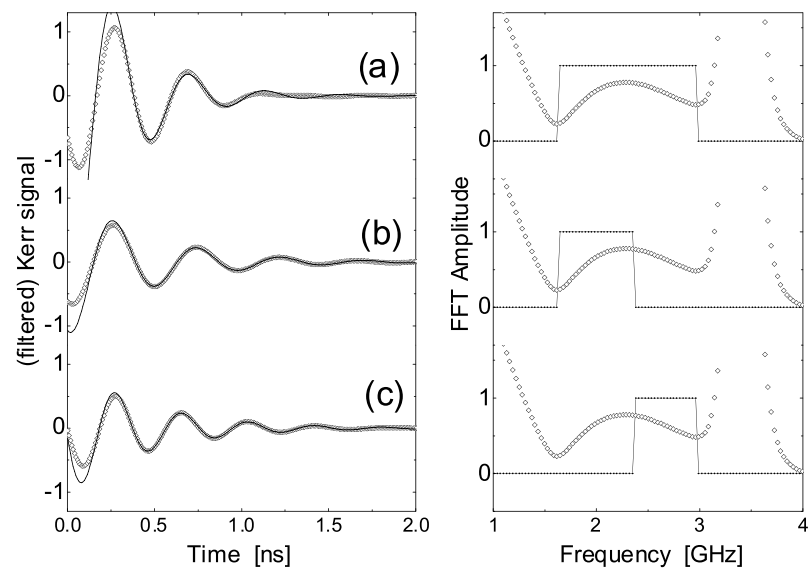

FIG. 3. Fourier filtering: Azimuthal mode separation. Detailed analysis of the azimuthal peak in Fig. 2(b). The left side shows the filtered signal (circles) with fit (solid line); the right shows the filters (dots) and FT of the original curve (circles). We find that the decay time for the whole peak in (a) $(0.31 \mathrm{~ns})$ is composed of the two components (b) and (c) with 0.44 and $0.45 \mathrm{~ns}$.

two overlapping peaks. This is still quite a small correction when compared to the longer decay times of the radial modes. And again we see the same trend on the modes $(2,0)$ and $(3,0)$ as in the experiment, which differ from the calculated values $\tau_{\alpha=0.008}^{\text {calc }}$ only within the uncertainty [Eq. (2)]. Since the LLG simulations use a single phenomenological damping parameter we can immediately rule out different damping of one mode over another as the cause for the extra observed damping in the low-frequency modes. Rather, we can see that, since energy is conserved and the global energy loss is governed by $\alpha$, mode-mode coupling must channel energy from the low-frequency modes towards the higher frequency modes. Scattering must be consistent with solutions to the nonlinear wave equation and energy conservation. (That is, scattering to higher frequency modes must also reduce the amplitude if the scattering is in one direction in $k$ space.)

We conclude that we have presented a method to extract the decay rates for different modes from a single average precession component. For the low-frequency modes we find an increased damping whose possible origin lies in mode conversion. For the radial modes, we find a behavior as expected from the linearized LLG equation. These two important features are closely reproduced in the micromagnetic simulation where the phenomenological Gilbert damping model is implemented with a single alpha. This demonstrates that for confined single-material ferromagnetic structures no additional theory of dissipation is necessary for exact reproduction of such intricate experimental results: The single-alpha straightforward LLG is all that is required to understand and explain the physics. These measurements are the first clear demonstration that mode-mode coupling plays a significant role in magneti- zation dynamics, and the data demonstrate how mode conversion could be construed as damping.

Financial support by the Schweizerische Nationalfonds, ETH Zurich, and the Deutsche Forschungsgemeinschaft through the Forschergruppe FOG370/2-1 and the priority program SPP1133 is gratefully acknowledged. We thank D. Weiss for access to his microfabrication facilities.

[1] E. Beaurepaire, J.-C. Merle, A. Daunois, and J.-Y. Bigot, Phys. Rev. Lett. 76, 4250 (1996).

[2] B. Koopmans, M. van Kampen, J. T. Kohlhepp, and W. J. M. de Jonge, Phys. Rev. Lett. 85, 844 (2000).

[3] W. K. Hiebert, A. Stankiewicz, and M. R. Freeman, Phys. Rev. Lett. 79, 1134 (1997).

[4] Y. Acremann et al., Science 290, 492 (2000).

[5] M. Buess et al., Phys. Rev. Lett. 93, 077207 (2004).

[6] A. Barman, V. V. Kruglyak, R. Hicken, A. Kundrotaite, and M. Rahman, Appl. Phys. Lett. 82, 3065 (2003).

[7] J. P. Park, P. Eames, D. M. Engebretson, J. Berezovsky, and P. A. Crowell, Phys. Rev. Lett. 89, 277201 (2002).

[8] S.-B. Choe et al., Science 304, 420 (2004).

[9] H. Stoll et al., Appl. Phys. Lett. 84, 3328 (2004).

[10] C. H. Back et al., Science 285, 864 (1999); I. Tudosa et al., Nature (London) 428, 831 (2004).

[11] W. K. Hiebert, L. Lagae, and J. DeBoeck, Phys. Rev. B 68, 020402(R) (2003).

[12] Z. Celinski and B. Heinrich, J. Appl. Phys. 70, 5935 (1991).

[13] U. Ebels, L. D. Buda, K. Ounadjela, and P. E. Wigen, in Spin Dynamics in Confined Magnetic Structures I, edited by B. Hillebrands and K. Ounadjela (Springer, New York, 2002).

[14] A. Yu. Dobin and R. H. Victora, Phys. Rev. Lett. 92, 257204 (2004).

[15] M. Belov, Z. Liu, R. D. Sydora, and M. R. Freeman, Phys. Rev. B 69, 094414 (2004).

[16] G. Gubbiotti et al., Phys. Rev. B 68, 184409 (2003).

[17] K. Y. Guslienko and A. N. Slavin, J. Appl. Phys. 87, 6337 (2000).

[18] M. Buess et al., Phys. Rev. B 71, 104415 (2005).

[19] J. Raabe et al., J. Appl. Phys. 88, 4437 (2000).

[20] R. Damon and J. Eshbach, Phys. Rev. 118, 1208 (1960).

[21] M. Buess, Y. Acremann, A. Kashuba, C. H. Back, and D. Pescia, J. Phys. Condens. Matter 15, R1093 (2003).

[22] In principle, the sum of the demagnetizing factors adds up to exactly one. In our approximation we set $N_{z}=1$ for a thin film disk and calculate $N_{r}$ as described in [5,21].

[23] http://llgmicro.home.mindspring.com/

[24] The micromagnetic simulations discretize space into $10.6 \mathrm{~nm} \times 10.6 \mathrm{~nm} \times 15.0 \mathrm{~nm}$ pixels in a $375 \times 375$ array, $4 \mu \mathrm{m}$ on a side. The saturation magnetization, exchange stiffness, gyromagnetic frequency, and damping constant are $860 \mathrm{emu} / \mathrm{cc}, 1.3 \mu \mathrm{erg} / \mathrm{cm}, 17.6 \mathrm{MHz} / \mathrm{Oe}$, and 0.008 , respectively. The equations of motion were integrated in $0.4 \mathrm{ps}$ steps from $t=0$ to $20 \mathrm{~ns}$. The data were stored for the entire array and for the average value of each magnetization component. 